\title{
Monde et historicité à partir de Patočka
}

\section{Claude Vishnu Spaak}

\section{OpenEdition}

\section{Journals}

Édition électronique

URL : https://journals.openedition.org/alter/1892

DOI : $10.4000 /$ alter.1892

ISSN : 2558-7927

\section{Éditeur :}

Association ALTER, Archives Husserl (CNRS-UMR 8547)

\section{Édition imprimée}

Date de publication : 1 novembre 2019

Pagination : 101-118

ISBN : 978-2-9550449-5-7

ISSN : 1249-8947

Référence électronique

Claude Vishnu Spaak, « Monde et historicité à partir de Patočka », Alter [En ligne], 27 | 2019, mis en ligne le 22 décembre 2020, consulté le 13 juin 2021. URL : http://journals.openedition.org/alter/1892 : DOI : https://doi.org/10.4000/alter.1892

Ce document a été généré automatiquement le 13 juin 2021.

Revue Alter 


\title{
Monde et historicité à partir de Patočka
}

\author{
Claude Vishnu Spaak
}

\section{Introduction : Le problème phénoménologique d'une historicité cosmologique}

1 Est-il possible pour une cosmologie phénoménologique - perspective théorique qui est celle de Jan Patočka - de prendre en charge la question de l'historicité, c'est-à-dire de penser quelque chose comme un devenir processuel $d u$ monde orienté selon un sens, voire même une téléologie ? Ce déploiement temporel ne serait pas simplement celui de la réalité humaine, mais un temps beaucoup plus long et englobant, omni-englobant même, au sein duquel l'historicité humaine - si tant est d'ailleurs qu'il y en ait une propre à l'humanité dans son ensemble, par-delà la diversité des cultures et des traditions - devrait être elle-même comprise comme un moment ou même une époque fondée dans ce devenir du monde. Dans cette procession temporelle l'historicité humaine ne serait qu'une étape, sans doute même pas la dernière dès lors que l'on refuse toute représentation anthropocentrique $\mathrm{du}$ monde, ce que préconise la philosophie asubjective de Patočka et plus généralement toute cosmologie phénoménologique. La question est donc de savoir si le monde dans son déploiement temporel manifeste une trame, un développement réglé ou ordonné, conformément d'ailleurs à l'étymologie de ce concept : le monde, cosmos, est l'ordonnancement réglé du multiple ; ce à quoi s'oppose le monde n'étant pas dès lors le néant, mais le chaos.

2 Afin de formuler le problème, il peut être utile de partir de cette citation de Patočka selon laquelle « c'est [le monde] qui fait que les phénomènes soient phénomènes, qui les amène à la déterminité, à la limitation et à la délimitation, qui leur donne d'avoir une charpente interne et externe; demeurant lui-même obscur dans le fond de son injonction ajointante, il amène tout à la lumière de l'apparaître $»^{1}$. Autrement dit, le monde est d'une part la structure de l'apparaître, l'horizon sur fond duquel les phénomènes ontiques (qu'ils soient de nature objective matérielle ou subjective 
conscientielle) peuvent apparaître. Mais d'autre part le monde est tout autant le principe ontogénétique par lequel les choses s'individuent, se constituent dans leur être même. Le monde à la fois comme structure de l'apparaître et comme principe ontogénétique, c'est là en réalité une seule et même dimension si tant est qu'être et apparaître sont rigoureusement identiques. Cette identité de la chose en soi et du phénomène est une thèse phénoménologique classique, mais elle ne pouvait trouver sa formulation appropriée que dans la perspective cosmologique, et non dans une perspective subjectiviste, car on ne voit pas par exemple comment la conscience pourrait avoir la charge de constituer non seulement le sens d'être des phénomènes, mais bien également ces phénomènes eux-mêmes quant à l'être. Husserl ne se risquait d'ailleurs pas à conférer à la conscience une prérogative cosmogonique. Il se contentait de prêter à la conscience la charge de constituer le seul sens des phénomènes, mais cette solution posait des difficultés pour une phénoménologie transcendantale subjective, en particulier celle de savoir comment les faits ontiques du monde se conforment aux essences se constituant dans la conscience ${ }^{2}$. La subordination de l'être au sens n'est au contraire possible que si l'être est déjà traversé par le sens, ce qui à son tour suppose que la constitution de sens soit une constitution d'être, une mise en sens ou une mise en ordre, un ordonnancement que seul le concept de monde permet de penser.

3 Dans la perspective d'une cosmologie phénoménologique le monde est donc la condition transcendantale du sens des phénomènes et la condition ontogénétique de leur individuation. Le monde est la totalité de ce qui est, non pas assurément totalité additive car alors le monde serait un simple individu contenant en lui tous les autres. Parce qu'un tel supra-individu générique présupposerait nécessairement, en tant qu'individu justement, un horizon de monde pour apparaître, s'ensuivrait une régression logique à l'infini de l'enchevêtrement perpétuel de mondes dans d'autres mondes, à la manière des poupées russes. Le monde n'est rien d'individuel, c'est-à-dire rien d'étant; il y a une différence cosmologique entre le monde et les étants intramondains, dont le principe remonte à Heidegger et sa fameuse différence ontologique entre l'être et l'étant, sauf que Patočka ou bien Fink militent pour une identification de l'être au monde ${ }^{3}$. La totalité qu'est le monde doit donc être comprise non pas comme la simple collection ou somme de ce qui est, mais l'horizon non étant sur fond duquel ce qui est peut apparaître. Plus encore, cet horizon n'est pas statique, il est dynamique pour autant que le monde fait être tout ce qui est. Or c'est justement ce caractère dynamique du monde, procès de mondification, que l'on veut interroger ici, à partir de cette considération élémentaire selon laquelle le procès de l'individuation est multiple, à la fois selon l'espace si tant est qu'il n'y a d'individus qu'en rapport avec d'autres individus; mais aussi selon le temps, puisqu'il n'y a d'individus que dans la durée par laquelle ils adviennent à partir de la rencontre ou de la dissolution d'autres individus antérieurs, et où ils persistent eux-mêmes durant le temps qui leur est imparti au sein d'un faisceau de déterminations tenues et «en-durées». C'est donc pourquoi l'on peut se poser la question de savoir si les procès cosmologiques d'individuation suivent une direction, si à travers la multiplicité et la profusion de l'activité productrice du monde un sens est à l'œuvre, thème d'une histoire cosmologique.

4 Toute la question est en même temps de savoir comment penser un tel sens: soit comme une processualité qui manifeste certes une unité à travers le foisonnement ontogénétique faisant advenir sans cesse des individus finis de par sa créativité infinie et surpuissante, mais une unité pourtant dépourvue d'un quelconque telos sous-jacent 
susceptible de l'orienter ${ }^{4}$. Selon une telle perspective la référence à l'historicité semblerait alors perdre d'emblée en pertinence ou du moins en importance, face à ce qui relèverait plutôt d'une création perpétuelle de nouveauté, un tourbillon contingent de significations surgissantes que le monde improvise en permanence. Une seconde perspective consiste à donner à ce sens créateur une orientation téléologique, en vue d'une finalité qui oriente le processus cosmologique tout le long de son ontogenèse, toute la question étant bien sûr dès lors de savoir en quoi peut consister la finalité de cette histoire pensée téléologiquement. Enfin, une troisième possibilité est de penser un sens négativement téléologique, celui d'un déclin ou d'une compromission par laquelle le monde s'abîme ou s'exténue - s'aliène - de plus en plus au sein de son développement ontogénétique. On explorera brièvement tour à tour chacune de ces trois perspectives, mais dans la mesure où l'enquête est de nature phénoménologique un fil conducteur s'impose : la question de l'historicité du monde doit impérativement prendre en compte le rapport entre le monde et celui qui parmi les étants du monde est capable en vertu de sa constitution ontologique de poser la question du monde et de son historicité, un étant qui n'est pas seulement l'observateur extérieur des processus mondains mais qui en est lui-même partie prenante puisque cet observateur appartient essentiellement au monde, il en est une concrétion. Autrement dit, la question de l'historicité cosmologique doit réfléchir à la place de cet individu exemplaire et paradigmatique que le monde fait advenir au sein de cette trame cosmologique (si tant est qu'il y en a une), à savoir l'homme, question de la place ou de "la situation de l'homme dans le monde ", pour reprendre le titre d'un essai de Max Scheler. En effet, à la différence notable de la cosmologie physique et du naturalisme matérialiste qui caractérise sa démarche, la cosmologie phénoménologique, en tant qu'elle est phénoménologique justement, refuse de réduire toute réalité mondaine à sa seule signification objective matérielle, mais elle prend en considération sa structure d'apparaître, renfermant la possibilité au moins en droit d'être dévoilée par une conscience subjective, conscience d'un étant intramondain ayant un statut différent de l'objectivité matérielle car elle implique la possibilité d'une ouverture à la totalité, ouverture spirituelle ou compréhensive qui situe en retour le questionnement cosmologique dans un cadre que méconnaît la science naturaliste, à savoir le cadre de la corrélation entre d'une part l'apparaître et d'autre part le destinataire subjectif de l'apparaître, destinataire qui est certes à l'instar de toute réalité ontique constitué par le monde, mais en un sens spécifique puisque cette constitution est celle d'un individu ayant la charge de faire paraître cela (le monde) qui l'individue et le manifeste déjà. En effet, le sujet n'est pas un simple étant intramondain. En tant qu'esprit il est l'ouverture à la totalité sur le mode du dévoilement. Il est ce par quoi un fait quel qu'il soit est susceptible d'être connu (et il est de ce point de vue la condition de possibilité de l'activité scientifique, y compris donc de la science physique elle-même). Le sujet est même l'attestation de l'irréductibilité du monde à la seule réalité physico-chimique, mais qu'il y a un Weltgeist ou bien, pour ne pas conférer au monde des traits qui ne conviennent qu'à la seule conscience, une forme-monde dont l'analyse ne peut en aucun cas s'épuiser dans une interrogation scientifique sur le modèle des sciences empiriques naturalistes. 


\title{
II. Patočka et l'historicité comme « évolution créatrice » : un héritage de Bergson?
}

Dans la page conclusive du Monde naturel comme problème philosophique, Patočka écrit :

\begin{abstract}
La métaphysique au sens propre, la philosophie authentique, c'est [...] la doctrine de la constitution. C'est là que s'ouvre à la philosophie un champ de recherches scientifiques rigoureuses, dont l'orientation n'est pas celle que s'imagine l'ontologie objectiviste. Il ne s'agit pas de dépasser le sujet, conçu comme obstacle à la connaissance parfaite du monde, mais au contraire de chercher dans le sujet même la loi de l'expérience qui donne naissance à la réalité sous toutes ses formes, dans toute la diversité des phénomènes. Telle est, en effet, une tâche (idéale) de la philosophie: effectuer dans le détail l'analyse avant tout de toute expérience humaine, chercher à en élucider les lois immanentes et à réaliser toutes les possibles opérations de variation qu'admettent ces lois ou structures. La seconde tâche, qui relève plutôt du style de la «métaphysique " reçue, est celle d'une histoire universelle. Le tout de l'histoire universelle englobe pour nous non seulement l'histoire de l'homme, mais celle de toute la création; ce serait une interprétation de tout le devenir du monde sur la base des structures fondamentales de la subjectivité possible, telles que dévoilées par l'analyse constitutive. La tâche d'une telle historisation de l'univers pourrait être désignée par le terme d'évolution créatrice. L'évolution créatrice que l'univers entier traverse dans l'activité constituante, jusqu'aux accomplissements suprêmes de la création consciente dans l'histoire humaine, est bien sûr une tâche surhumaine, qui présuppose avant tout des progrès bien plus soutenus dans les sciences et un travail analytique philosophique lui aussi plus intense, seule l'action conjuguée de l'un et des autres pouvant venir à bout de la tâche d'interpréter toute existence à partir des sources internes de la vie même ${ }^{5}$.
\end{abstract}

6 Ce texte est intéressant d'abord parce qu'il appelle à une réconciliation ou même une unification entre d'une part les sciences objectives de la nature et d'autre part la philosophie en sa visée spéculative la plus haute, à savoir la métaphysique ${ }^{6}$. Cette réconciliation est elle-même présentée comme une tâche historique consistant à dépasser la situation dans la modernité de séparation des discours et des méthodes scientifiques, due à la spécialisation des savoirs, mais aussi au hiatus entre le champ du spirituel et le champ matériel, hérité pour partie du dualisme cartésien des substances, res extensa et res cogitans. Patočka, fidèle à sa modestie philosophique, présente cette tâche historique de réconciliation et d'unification de la science et de la philosophie comme surhumaine pour le moment, en attente de progrès à venir dans les sciences mais aussi dans la philosophie. La difficulté provient typiquement du fait qu'une histoire universelle implique de se prononcer sur une situation dans le devenir du monde qui préexistait à la corrélation entre le sujet et l'apparaître, puisque cette histoire universelle est précisément censée rendre compte de l'émergence de la corrélation ellemême ${ }^{7}$. Autant dire que l'histoire universelle doit consentir à sortir de la phénoménologie, non pas en atteignant d'hypothétiques réalités en soi et non apparaissantes, mais en débusquant les conditions dernières de l'apparaitre. Il s'agit d'une histoire "de toute la création", "interprétation du devenir du monde" renvoyant le développement des ontologies matérielles ainsi que des structures de la subjectivité, au monde comme à leur origine constituante conjointe. Le monde, ici, semble même en sa générativité pouvoir être identifié à la vie, puisqu'il s'agit comme le dit Patočka « d'interpréter toute existence à partir de la vie même $»^{8}$. 
7 L'assimilation du cosmos à une phusis intégrale rappelle le geste philosophique bergsonien à l'œuvre dans L'évolution créatrice, que Patočka évoque tout à fait explicitement ici. Pouvons-nous donc trouver dans le bergsonisme la clé de compréhension d'une histoire cosmologique? Rien n'est moins sûr, si tant est que Patočka reproche à Bergson une représentation toute psychologique (et donc ontique) de l'élan vital et du mouvement qui le caractérise comme durée, durée qui est comprise par Bergson sur le modèle de la conscience qui, par une inflation métaphysique, en vient à s'appliquer à toute réalité. Même la matière, dans certains passages de L'évolution créatrice, semble chez Bergson reconductible, par-delà tout dualisme espritmatière, à ce processus moniste de l'élan vital, entendu comme un mouvement créateur spirituel, et dont la matière ne serait qu'une production ou une procession pensée sur un mode crypto-plotinien, puisque la matière n'est «qu'un simple arrêt de l'action génératrice de la forme ${ }^{9}$, elle est issue du « courant vital » et se résume à des " parties congelée de sa substance qu'il charrie le long de son parcours $»^{10}$. Bergson, qui demeure aux yeux de Patočka prisonnier des métaphores ontiques, dont nous avons ici un exemple, substantialise l'élan vital - c'est-à-dire le monde lui-même - et ne parvient donc pas à penser la différence cosmologique entre d'une part le monde comme ouverture, et d'autre part les étants intramondains (matériels ou conscientiels) qu'il fait advenir. Bergson appartient donc encore selon Patočka à l'histoire de la métaphysique, au sens heideggérien des figures époquales où la question de l'être est confondue avec la question de l'étant :

[Bergson] s'efforce de résoudre métaphysiquement des questions qui demandent à être développées en premier lieu de façon descriptive et analytique [...]. Les images ontiques de Bergson occultent la nécessité que la mise à découvert des choses soit médiatisée par autre chose en quoi seul les choses sont des choses à découvert ${ }^{11}$.

8 Cette « autre chose » censée médiatiser la mise à découvert des choses, et qui n'est pas elle-même une chose, est évidemment le monde. Mais en admettant que l'on résiste à une telle substantialisation du monde (ou bien ce qui revient au même: à une psychologisation du mouvement mondifiant), comment comprendre la proposition de Patočka selon laquelle l'histoire universelle du monde n'est autre que celle de la vie, monde et vie étant ici présentés par le philosophe tchèque comme synonymes? La formation des galaxies, des étoiles, des planètes, toutes choses que le sens commun informé par des connaissances élémentaires en sciences de la nature conçoit comme des préalables à l'apparition de la vie, elle-même une exception ou un épiphénomène à l'échelle cosmique, sont-elles aussi des phénomènes de la vie? La vie est-elle omnienglobante de toute réalité ? Mais si tel est le cas, n'est-ce pas là somme toute une position voisine de celle de Bergson, moyennant la simple mention qu'il ne faut pas confondre cette vie avec du vivant (ce que ne manque pas de faire Bergson lui-même au demeurant, lorsqu'il oppose sans relâche la vie se faisant au "tout fait » des êtres vivants, tous traversés par cet élan vital qui ne se réduit pas à un vivant quelconque)?

Il faut ici rendre compte d'une autre différence essentielle entre Patočka et Bergson: lorsque le philosophe tchèque assimile le monde à une vie, il faut l'entendre en un sens plus aristotélicien que bergsonien, au sens de la phusis plutôt que de la zoe ou du bios : le monde comme vie est un mouvement universel qui se pluralise infiniment en des processus ontogénétiques diversifiés. Or chez Aristote la phusis n'est pas assignable à un simple élan vital. Aristote est déjà phénoménologue selon Patočka, car il voit que le mouvement ontogénétique qui procède de l'être (phusis) a pour sens l'apparaître : 
Le dévoilement de la chose dans sa figure véritable est le mouvement propre de la chose qui sort du retrait pour se déployer dans la plénitude de sa présence et sombrer de nouveau dans l'absence. C'est dans cette connexion qu'Aristote envisage les choses. La manifestation de l'être est pour lui manifestation de la chose. La chose se manifeste par où elle est, sa manifestation est un mouvement. Aussi faut-il comprendre les choses dans leur mouvement ${ }^{12}$.

10 À défaut de penser l'élan vital sous cette forme phénoménologique, Bergson le comprend dans l'horizon exclusivement pratique d'un accroissement, ce qui rend difficile de penser la dimension qui chez l'homme deviendra l'ouverture compréhensive et pour tout dire désintéressée à l'apparaître sur le mode du dévoilement, ce que thématise Patočka au titre du troisième mouvement de l'existence humaine. Au lieu de cela, Bergson développe une conception de l'homme dans laquelle la connaissance est entrelacée avec les intérêts pratiques et même techniques de l'existence, centrée sur l'intelligence et dans une moindre mesure l'intuition, laquelle chez l'homme est moins développée sur le plan biologique évolutif que l'intelligence (« dans l'humanité dont nous faisons partie, l'intuition est à peu près complètement sacrifiée à l'intelligence $»^{13}$ ). L'idée qu'a Bergson de l'homme, qu'il n'hésite pas d'ailleurs à présenter comme la fin de la création (mais non son point culminant, étant donné que la vie peut parcourir des chemins différents vers la croissance et l'adaptation, ce qui rend la configuration humaine toute contingente), c'est celle d'un homo faber plus que d'un homo sapiens ${ }^{14}$, si l'on entend par celui-ci un sujet ouvert sur le plan théorique à la totalité selon la modalité suprême de la connaissance pour la connaissance, et sur le plan pratique à la communauté moralisée des êtres composant un royaume des fins au sens kantien. La supériorité de l'homme réside en ceci chez Bergson que "l'homme peut faire ce qui lui plait» en "ayant sauté l'obstacle de la matière ». Mais sauter l'obstacle signifie simplement : croître de façon moins entravée, sur le plan non plus de la vie mais de la technique. Bergson ne voit pas que le monde, s'il aspire à quelque chose, c'est moins à la croissance qu'à la manifestation, voire même peut-être à l'auto-manifestation.

Chez Patočka l'histoire cosmologique mais aussi humaine (puisque l'homme est un "enfant du monde» pour reprendre l'expression de Husserl) ne peut avoir le sens d'une simple croissance perpétuelle, justement parce que le troisième mouvement de l'existence l'emporte chez l'homme sur les deux premiers (notamment le second: mouvement par lequel l'homme se caractérise comme homo faber, et où l'homme n'exprime justement pas encore sa potentialité la plus haute ${ }^{15}$ ). La conception bergsonienne de l'éthique comme "supplément d'âme » pour le développement de la technique (elle-même vouée à servir le progrès moral de l'homme, c'est-à-dire sa libération à l'égard de la matière ${ }^{16}$ ) affaiblit sa portée pour n'en faire qu'une sorte de variable d'ajustement de la technique, quand bien même Bergson défend en même temps la relation réciproque: la technique est là pour servir l'âme, «la mystique appelle la mécanique ». Reste que chez Patočka, à la différence de Bergson, les deux plans (spéculatif pour l'éthique, instrumental pour la technique) sont foncièrement hétérogènes l'un à l'autre ${ }^{17}$.

12 En définitive, on peut même raisonnablement penser que chez Bergson il n'y a pas vraiment de place pour une histoire cosmologique, si tant est que l'élan vital, cette poussée créatrice de l'être, ne suit aucune trajectoire particulière si ce n'est celle de la croissance aveugle, multidimensionnelle et polyphasée à partir du fonds préindividuel (apeiron), si l'on reformule l'idée dans le langage de Simondon ${ }^{18}$. Alors que l'histoire 
suppose la souscription à l'idée d'une vectorisation temporelle, Bergson recourt moins à la métaphore de la ligne qu'à celle d'une onde qui se propage en cercle autour d'un centre : « De notre point de vue, la vie apparaît globalement comme une onde immense qui se propage à partir d'un centre et qui, sur la presque totalité de sa circonférence, s'arrête et se convertit en oscillation sur place $»^{19}$.

13 «En un seul point», ajoute pourtant Bergson, «l'obstacle a été forcé, l'impulsion a passé librement. C'est cette liberté qu'enregistre la forme humaine $»^{20}$. L'homme peut bien être posé comme le terme ou le but de l'évolution, mais à condition selon Bergson que l'on ne comprenne pas par là que « l'humanité telle que nous l'avons sous les yeux [soit] préformée par le mouvement évolutif $»^{21}$. En effet, "l'évolution s'est accomplie sur plusieurs lignes divergentes $»^{22}$.

\section{L'institution originaire des idéalités et la tâche historique infinie de leur élucidation dans la conscience (Husserl)}

14 L'évocation de Bergson permet par la négative de comprendre qu'une phénoménologie cosmologique, pour autant qu'elle prend au sérieux l'idée d'une historicité universelle, doit rendre compte d'une des visées de cette historicité, voire même sa visée la plus haute, qui est le développement de la corrélation entre l'apparaître et le sujet s'y rapportant (quand bien même cette visée ne serait pas consciente, précisément parce que la conscience procède de la corrélation une fois engendrée). C'est donc ainsi dans un second temps l'idée de ce qu'il faut bien appeler une histoire téléologique que l'on explorera, dont la finalité serait pour le monde de faire émerger la corrélation. Sur cette conception téléologique Patočka doit sans doute beaucoup à Husserl. À la même époque en effet où Patočka écrit Le Monde naturel comme problème philosophique, texte dont nous avons commenté la conclusion, Husserl développait le thème de l'historicité autour de 1936 dans la Krisis ou bien dans sa fameuse Conférence de Vienne. L'historicité chez Husserl a le sens d'une tâche de la raison dont la prise de conscience a lieu dans le monde grec, par une institution originaire (Urstiftung) dans une Europe considérée moins comme concept géographique ou culturel, que comme concept spirituel ${ }^{23}$. Cette tâche de la raison est portée ensuite par la communauté des hommes de science qui se reconnaissent autour de l'idéal d'atteindre les essences idéales (nécessaires et universelles) ou structures de l'apparaître. La conscience de cette tâche (qui n'est autre que celle d'élucider les structures de la corrélation entre les essences de l'apparaître et ses modes subjectifs de donnée) est advenue lorsque les Grecs ont découvert d'abord dans la science mathématique et concomitamment dans la philosophie comme discours portant sur des idéalités inconditionnées, un champ de questionnement ayant pour thème le domaine de l'absolu, par-delà le monde de la vie (Lebenswelt) où ils trouvaient jusqu'alors les significations nécessairement particulières de leur existence, autour de leurs croyances, traditions, mythes, etc. L'élévation de la conscience vers l'intuition des essences absolues se fait certes chez Husserl sur le plan de la conscience individuelle, au terme du processus méthodique de la mise entre parenthèse ou epoché de l'attitude naturelle et de sa thèse naïve de l'évidence du monde et des réalités intramondaines, puis par l'effectuation de la réduction phénoménologique qui consiste à reconduire les significations de toutes les objectités mondaines à leurs sources constituantes dans la conscience qui n'est elle-même rien de mondain, mais la condition transcendantale du 
sens des réalités mondaines, matérielles tout autant que psychiques. Mais cette méthode phénoménologique elle-même n'est praticable sur le plan de la conscience individuelle qu'à un certain moment de l'histoire collective du développement de la raison, qui trouve ses origines instituantes dans le monde grec et traverse des étapes successives, où des thèses philosophiques unilatérales se doivent d'être dépassées (notamment, dans la modernité, l'objectivisme, l'empirisme, le transcendantalisme naif), avant de s'accomplir enfin dans une position philosophique satisfaisante qui est justement la phénoménologie transcendantale.

15 À l'évidence la cosmologie phénoménologique de Patočka ne peut pas suivre Husserl sur son orientation générale selon laquelle l'absolu est identifiable à la conscience constituante. L'absolu phénoménologique tout autant qu'ontogénétique ne peut être comme nous l'avons dit que le monde lui-même, auquel la conscience appartient, elle qui n'est jamais que le mode d'être d'un étant intramondain et relatif, contrairement à ce que voulait démontrer Husserl, en distinguant entre différents plans de la conscience (le plan psychique intramondain dissocié du plan transcendantal spirituel acosmique). Mais l'on pourrait s'attendre également à ce que Patočka critique Husserl sur un autre point également, à savoir la thèse husserlienne de l'institution originaire des idéalités dans la conscience puis leur élucidation progressive au sein d'une histoire relevant exclusivement de l'humanité (et même d'une certaine culture: celle des Grecs), comme s'il n'y avait d'historicité que pour et par le sujet humain. Si l'historicité trouve sa source de déploiement dans le monde lui-même, alors ce n'est pas l'homme qui institue les tâches de la raison : il n'y a d'institution originaire des tâches infinies de la raison que par l'événement inaugural au sein duquel le monde entame son procès d'ordonnancement de tout ce qui est, ordonnancement au sein duquel l'historicité humaine ne peut plus apparaître que comme un développement tardif.

Malheureusement Patočka ne s'aventura jamais très loin dans cette perspective philosophique, peut-être comme évoqué plus haut en raison de sa modestie face à des tâches jugées "surhumaines". Mais ce qui est plus problématique, par une sorte de ralliement tardif à Husserl et plus encore à Heidegger, Patočka dans les Essais hérétiques se range au présupposé selon lequel l'historicité concerne la seule relation entre le Dasein et le monde. On voit mal à lire les Essais hérétiques comment il peut y avoir d'histoire précédant l'émergence de la Bewusstsein ou du Dasein. Qui plus est, l'historicité est même reversée non pas du côté de l'humanité en général, mais d'une certaine humanité culturellement située, capable de se hisser hors de l'attachement au monde naturel, afin de tourner son regard vers le monde en totalité, et l'éclairer sous le jour de la problématicité. Cette aptitude philosophique au dévoilement du monde en tant que monde, devient même chez Patočka un marqueur permettant de discriminer entre les humanités historiques (l'humanité européenne essentiellement) et les humanités "préhistoriques": des peuples qui sont certes dans l'humanité du point de vue des exigences anthropologiques et des pratiques symboliques associées (langage, systèmes de croyance, traditions, art, religion, mythes, etc.), mais qui ne se sont pas élevés au plan de la spiritualité sous sa forme la plus vive, philosophiquement tournée sur le monde comme problème, problème qui seul peut émerger de l'étonnement philosophique (thaumazein) face à la présence de l'étant en tant qu'étant, donc au sein d'une expérience que les Grecs typiquement firent et nous léguèrent. Cela ne signifie pas que le troisième mouvement de l'existence humaine, comme Patočka le nomme (mouvement de percée vers le monde en totalité), ne soit pas toujours déjà là en toute 
humanité ; il est bien là, mais sur le mode de la latence, et si le monde se donne bien à voir en totalité chez les humanités préhistoriales, c'est seulement sur le plan du mythe, qui révèle certes la vérité de l'être comme le soutient Patočka notamment dans Platon et l'Europe, mais qui ne tire pas les implications de ce dévoilement, puisque le mythe enjoint encore les peuples anhistoriques à la soumission et à l'acceptation de l'ordre cosmique fixe et imposé, dont les Dieux sont les garants.

\section{Panthéisme et devenir historial du monde (Scheler)}

La question que l'on pourrait poser dès lors est celle de savoir si l'on peut réconcilier la perspective programmatique annoncée par Patočka à la fin de sa thèse d'habilitation de 1936 d'une histoire universelle du monde en totalité, avec sa conception effective de l'historicité, qui l'inscrit dans le giron exclusif de la réalité humaine. Comment effectuer la médiation entre d'une part l'historicité humaine, pensée téléologiquement comme mouvement d'accomplissement de la corrélation dans la vie philosophique, et d'autre part l'histoire du monde, où l'homme représente dès lors le moyen par lequel le processus cosmologique atteint à l'auto-manifestation par le truchement et l'intercession de l'humanité ? Pour répondre à cette question, on peut se tourner vers Max Scheler, que Patočka connaissait bien et dont il avait lu son opuscule sur La situation de l'homme dans le monde. Scheler abonde lui aussi dans le sens d'une cosmologie, seulement chez lui elle prend les allures d'un panthéisme dynamique. Selon Scheler, la divinité est le principe immanent du monde qui cherche à se réaliser en lui, ce qui n'est possible que dans le cours de l'histoire universelle qui voit émerger l'esprit graduellement en l'homme :

Le principe des choses, pour réaliser sa deitas, l'abondance d'idées et de valeurs immanentes à celle-ci, devait libérer l'impulsion créatrice pour s'accomplir lui-même dans le déroulement temporel du processus universel, - il devait s'accommoder pour ainsi dire de ce processus, pour actualiser en lui et par lui sa propre essence. L'« Être-par-soi » ne parvient vraiment à l'existence divine que dans la mesure où, en l'impétueux mouvement de l'histoire universelle, il réalise en l'homme et par l'homme la deitas universelle. Et ce processus [...] ne peut approcher de son but, qui est l'accomplissement propre de la divinité, que dans la mesure où le monde lui-même sera devenu l'incarnation achevée de l'esprit et l'impulsion éternels. C'est seulement dans le mouvement de cette énorme tempête qu'est le "monde » qu'une adaptation réciproque peut se produire entre l'ordre des formes et des valeurs, et les puissances réellement agissantes ${ }^{24}$.

Il n'est pas possible de commenter toutes les implications de ce texte très spéculatif, et qui ne doit pas conduire à minorer l'attention toute méticuleuse avec laquelle Scheler, dans un dialogue constant avec les sciences expérimentales de la nature de son temps (physique et surtout biologie), articule les différents jalons de cette histoire universelle tempétueuse du monde en s'appuyant sur les découvertes de la science positive: lois physiques du monde, lois statistiques qui permettent de rendre compte de la réunion des conditions nécessaires à l'apparition de la vie; puis, sur le plan de la vie, les différentes étapes d'une évolution biologique adaptative : poussée affective chez les végétaux, instinct chez l'animal, mémoire associative, intelligence pratique chez les animaux supérieurs, et puis enfin l'épanouissement de l'esprit proprement dit qui, à la différence de toutes les prestations communes aux êtres vivants, consiste à se rapporter non plus à un milieu local aux fins de la survie, mais au monde lui-même. Comme l'écrit Scheler dans un texte anticipant sur Patočka : 
Le caractère fondamental d'un être «spirituel » est son détachement existentiel, sa liberté, la possibilité qu'il a - ou qu'a du moins le centre de son existence - de se dégager de la fascination et de la pression de ce qui est organique, de se rendre indépendant de la « vie » et de tout ce qui relève de la « vie », par conséquent aussi de sa propre intelligence soumise aux tendances. Un tel être spirituel n'est plus assujetti au désir ni lié au milieu, il est « libéré du milieu », nous dirons qu'il est « ouvert au monde ", qu'il a un « univers ${ }^{25}$.

On laissera de côté le problème de savoir si l'assimilation du principe du monde à la deitas ne risque pas de faire succomber Scheler au même piège que Bergson, celui de ne pas prendre en compte la différence cosmologique dont nous avons parlé. La deitas, qui est la susbstance spirituelle du monde en demande de réalisation dans l'histoire universelle, est certainement pensée par Scheler sur un mode ontique, comme un sujet omni-englobant, une conscience ensommeillée tout d'abord, et ne se réveillant pleinement à soi-même qu'avec l'émergence de l'homme et la reconnaissance par celuici de sa propre essence spirituelle. On ne traitera pas non plus le problème de savoir si l'assimilation de la deitas au principe du monde ne génère pas une forme cryptée de transcendance de ce principe divin en attente de réalisation dans l'historicité cosmologique, et si Scheler de la sorte ne réintroduit pas subrepticement une forme d'acosmisme. Enfin, on ne dira rien de l'anthropocentrisme ontologique à l'œuvre dans la pensée de Scheler, hérité sans doute de l'importance chez lui du motif chrétien de l'essence de l'homme constituée à l'image et à la ressemblance de Dieu. Si Scheler a rompu avec à peu près tout de la théologie chrétienne, son panthéisme historial conserve tout de même intacte l'idée selon laquelle l'homme est la fin et le terme de la création. On a noté au contraire que dans une perspective asubjective qui est celle de Patočka, l'anthropocentrisme ontologique d'un Scheler est irrecevable. Mais un tel anthropocentrisme n'est-il pas inéluctable si l'on souscrit à l'idée que l'histoire universelle est indexée sur le développement de l'esprit, c'est-à-dire (pensé en termes phénoménologiques) le développement de l'a priori de la corrélation par quoi un sujet advient qui, en son être, occupe la position de destinataire de l'apparaître, c'est-à-dire destinataire du monde comme apparaître ? Une solution existe pourtant, afin d'éviter la conséquence anthropologiste de cette historicité cosmologique à caractère téléologique, tout en maintenant l'idée que le telos visé par cette histoire est celui d'une auto-révélation de l'apparaître à lui-même (le monde aspirant en quelque sorte à s'auto-comprendre). Pour cela il suffit de purger cette approche spéculative de tout reste de présuppositions théologiques : ce n'est pas l'homme qui est appelé par la deitas à lui servir de miroir (l'homme "crée à l'image et à la ressemblance de Dieu »); c'est plutôt le Dasein (l'étant qui en son être est ouvert à l'être) qui est ainsi appelé, or le Dasein peut s'appliquer à tout étant spirituel existant dans l'univers. De ce point de vue l'on dira donc qu'il était contingent que tel étant-ci advienne (le sujet humain en l'occurrence), mais il était toutefois nécessaire qu'un étant spirituel finisse par émerger dans l'histoire (ou une multitude, si l'on endosse l'idée qu'il existe des êtres extraterrestres), capable de se poser comme sujet ouvert compréhensivement au monde, de porter au paraitre le monde lui-même qui fait tout paraître, et d'ériger cet apparaître au rang de problème dans la vie philosophique. 


\section{Conclusion : la dialectique négative de l'historicité du monde (Heidegger, Adorno)}

Dans ce qui a précédé nous avons tâché de reconstruire la position philosophique de Patočka (ou bien une certaine position, compte tenu des revirements de ce penseur au cours de sa carrière) sur la question de l'histoire cosmologique. Pour ce faire, nous avons cherché à clarifier sa position en la confrontant tour à tour à celles de Bergson, Husserl et Scheler (et de manière plus furtive à Heidegger). Nous terminerons ces analyses sur une remarque critique à l'égard de la conception patočkienne, nous permettant d'esquisser une troisième voie pour rendre compte du sens d'une historicité du monde. Il y a dans la conception téléologique d'un devenir historique du monde, que ce soit la téléologie non anthropocentrée de Patočka ou bien la téléologie anthropocentrée de Scheler, un présupposé spéculatif hérité de l'idéalisme allemand (on pense à Hegel, Schelling, Schiller, Fichte et déjà Kant), lui-même inscrit dans une certaine configuration de pensée à l'œuvre dès le siècle des Lumières, selon lequel l'histoire (humaine ou universelle) est le théâtre du devenir progressif de l'esprit vers ses deux formes les plus hautes : la vie dans la vérité par la connaissance de l'absolu et la vie dans le bien par la réalisation de l'absolu dans la communauté moralisée des êtres spirituels. Sur ce point Patočka, dans le fond, importe à la suite de Husserl dans la tradition phénoménologique cet optimisme, quand bien même il le problématise (non moins que Husserl d'ailleurs) en soulignant le caractère tragique, voire polémologique, de cette historicité de l'esprit dans sa conquête difficile et douloureuse de son ouverture méditante sur le monde ${ }^{26}$. Face à cela, on peut songer à une autre voie interprétative de l'histoire universelle, une voie plus pessimiste sans doute, consistant à douter de l'accomplissement historique de la spiritualité dans ses formes supérieures.

21 En effet, il convient peut-être de profiter de notre situation époquale présente, marquée par la dévastation écologique de notre planète et l'affaissement de notre monde de plus en plus incapable de faire sens, afin de se demander si la promesse d'une époque finale enfin marquée par le triomphe de l'esprit (ou bien dont l'atteinte se ferait à l'infini, dans un mouvement de convergence asymptotique), est encore audible lorsque l'on réfléchit à la situation de l'homme dans le monde non seulement après la fin de l'histoire de la métaphysique (identifiée au $\mathrm{XX}^{\mathrm{e}}$ siècle), mais si l'on peut dire après la fin de la fin de la métaphysique dans le contexte contemporain, intensément voire même exclusivement dominé par la technique. Peut-être que Bergson avait raison finalement de conférer à la figure de l'homo faber une place importante, à ceci près qu'elle ne serait pas l'essence de l'homme, mais sa contre-essence, une fois refoulée son essence pensante (le Dasein) qui dans l'histoire se trouve de plus en plus réprimée. Il faut dès lors se demander si l'a priori de la corrélation, si cher à la phénoménologie, en admettant qu'il soit advenu et se soit réalisé à un certain stade de l'histoire cosmologique, ne court pas de plus en plus le risque de disparaitre. La question de la suppression du sujet de la corrélation, ce sujet enraciné dans l'humain mais qui en soi n'est rien d'humain (là était d'ailleurs la thèse de Heidegger contre Sartre dans sa "Lettre sur l'humanisme »), doit se poser à l'heure de l'ère post-métaphysique marquée par la technique et la toute-puissance de la raison instrumentale, qui ayant pris le pas sur les formes plus hautes de la spiritualité (contemplatives, pratiques, esthétiques), dissout de plus en plus dans sa logique totalitaire l'altérité des objets ravalés au seul plan de la matière devenue stock d'exploitation, ainsi que des sujets démis de leur 
essence pensante pour être transformés en producteurs/consommateurs. La question est de savoir si l'a priori de la corrélation est un a priori historique, non pas au sens foucaldien qui le confinerait trop étroitement dans une époque particulière (renaissance, âge classique, modernité) ${ }^{27}$, mais un a priori historial, qui a pour cadre le déploiement de la métaphysique depuis les Grecs jusqu'à nous. Une réflexion philosophique sur l'historicité du monde, prenant en compte sa propre situation (en tant que réflexion, donc en tant qu'elle émane d'une instance subjective réfléchissante), doit donc tout autant penser les conditions sous lesquelles émerge la corrélation entre l'apparaître et son destinataire, mais également celles de la destruction imminente de cette corrélation, et ce non pas seulement au vu de la catastrophe écologique en cours qui menace les écosystèmes et la survie de l'homme, mais plus profondément encore face à la déshumanisation rampante de l'homme lui-même face à l'emprise de la technique.

C'est ce qu'indiquait Heidegger dans des pages importantes des Contributions à la philosophie, lorsqu'il développait les implications du concept de «machination" (Machenschaft). C'est aussi ce qu'Adorno pensa comme dialectique négative ${ }^{28}$, ou raison totalitaire ${ }^{29}$ dont le déploiement historique implique inévitablement la dissolution des individus et l'exploitation intégrale de la nature au sein d'une domination purement instrumentale, au mépris des formes supérieures de la raison spéculative, qui se dévoile de plus en plus au fil de cette dialectique n'avoir été qu'un leurre ou un narcotique, comme si la croyance en la transcendance et dans l'advenue d'un règne spirituel non concerné par les tribulations de la matière représentaient une illusion utile dont l'histoire s'était nourrie tout le long de son déploiement pour mieux occuper les hommes et les mettre au travail pour servir cette raison instrumentale vorace et tentaculaire... C'est alors un toute autre sens de l'histoire universelle qu'il nous faudrait penser, accessible pour la première fois depuis notre époque post-métaphysique où les masques et les mirages de la raison spéculative se sont écroulés.

\section{NOTES}

1. J. Patočka, « Le tout du monde et le monde de l'homme. Remarques sur un essai contemporain de cosmologie ", in Le monde naturel et le mouvement de l'existence humaine, trad. par E. Abrams, Dodrecht, Boston, Londres, Kluwer, 1988, p. 268.

2. Fink a soulevé cette difficulté à de nombreuses reprises. Ainsi : « Husserl n'en vient nulle part à une détermination ontologique du procès constitutif [...]. Chez Husserl le sens de la 'constitution transcendantale' oscille entre formation de sens et création » (E. Fink, «L'analyse intentionnelle et la pensée spéculative ", in Proximité et distance, trad. par J. Kessler, Grenoble, Millon, 1976, p. 124).

3. Pour un traitement de cette question sous l'angle de la pensée de Fink, voir aussi la contribution d'o. Stanciu dans le présent recueil, intitulé «Le méontique et le monde. Sur l'itinéraire philosophique d'Eugen Fink». Ainsi que l'écrit Fink: "L'être lui-même n'est pas l'universel flottant dans le vague, il est l'espace-temps du monde, qui comprend tous les étants » (E. Fink, op. cit., p. 126). 
4. C'est dans cette perspective que D. Duicu interprète la pensée de Patočka dans «Le déplacement de la question téléologique dans la philosophie phénoménologique de Patočka ", Revue de métaphysique et de morale, 2017/3 ( $n^{\circ}$ 95), p. 341-356. D. Duicu voit en effet dans la téléologie le reste d'une "ancienne orientation métaphysique que l'on pouvait croire définitivement révolue » (p.342) et à laquelle Patočka échappe. Il nous semble que l'on peut adopter une attitude téléologique qui soit non métaphysique, à condition de respecter certains principes fondamentaux de la méthode phénoménologique, dont la différence ontologique entre le monde et les étants apparaissants. En ce sens nous voyons plus volontiers dans la pensée de Patočka les bases d'une téléologie renouvelée même si nous souscrivons aux craintes de D. Duicu concernant les dangers d'une téléologie naïve référant l'histoire à un projet émanant d'une instance ontique (esprit, divinité, etc.).

5. J. Patočka, Le monde naturel comme problème philosophique, trad. par E. Abrams, Paris, Vrin, 2016, p. $167-168$.

6. C'est là aussi le projet de Husserl, celui d'unifier les sciences de la nature et les sciences de l'esprit considérées comme les deux ramifications d'une seule et même science universelle du monde en tant qu'universum, tâche qui n'est autre que celle de la philosophie elle-même comme science rigoureuse. Cf. E. Husserl, Nature et esprit (1927), trad. par J. Farges, Paris, Vrin, 2017.

7. La philosophie vise à penser rien de moins que l'origine du monde: «La philosophie ne cherche pas simplement une image du monde, ni une forme pure; elle se met en quête de l'origine $\mathrm{du}$ monde au sens radical. Elle est historique non seulement parce qu'elle s'intéresse aux changements que subit le monde, mais aussi, éminemment, parce qu'elle en interroge l'essence et l'origine » (J. Patočka, «Quelques remarques sur le concept d'“histoire universelle" », in L'Europe après l'Europe, trad. par E. Abrams, Lagrasse, Verdier, 2007, p. 168-169.

8. Nous renvoyons sur ce point aux travaux de F. Jacquet sur la pensée de la phusis chez Patočka, notamment dans F. Jacquet, Patočka. Une phénoménologie de la naissance, Paris, CNRS éditions, 2016. Voir aussi P. Rodrigo, "Symphysis. Patočka face à 'l'empirie trop grossière et naïve' d'Aristote ", in C. V. Spaak, O. Stanciu (éds.), Jan Patočka. Métaphysique et phénoménologie, Paris, Le Cercle Herméneutique, 2014.

9. H. Bergson, L'évolution créatrice, Paris, PUF, $12^{\mathrm{e}}$ édition, 2013, p. 240.

10. Idem.

11. J. Patočka, «Supplément à la $2^{\mathrm{e}}$ édition tchèque (1970) à : " Le monde naturel dans la méditation de son auteur trente-trois ans après", in Le monde naturel comme problème philosophique, op. cit., p. 204-205.

12. J. Patočka, Platon et l'Europe, trad. par E. Abrams, Lagrasse, Verdier, 1983, p. 203.

13. H. Bergson, L'évolution créatrice, op. cit., p. 267-268.

14. "Si nous pouvions nous dépouiller de tout orgueil, si, pour définir notre espèce, nous nous en tenions strictement à ce que l'histoire et la préhistoire nous présentent comme la caractéristique constante de l'homme et de l'intelligence, nous ne dirions peut-être pas Homo sapiens, mais Homo faber. En définitive, l'intelligence, envisagée dans ce qui en paraît être la démarche originelle, est la faculté de fabriquer des objets artificiels, en particulier des outils à faire des outils, et d'en varier indéfiniment la fabrication", H. Bergson, L'évolution créatrice, op. cit., p. 140.

15. Cf. J. Patočka, «Méditation sur "Le monde naturel comme problème philosophique" ", in Le monde naturel et le mouvement de l'existence humaine, op. cit., p. 50-127 (en particulier p. 107-125).

16. Cf. H. Bergson, Les deux sources de la morale et de la religion, Paris, PUF, 1984, p. 329-331.

17. Sur la place de l'éthique chez Patočka, irréductible à la logique instrumentale, voir $\mathrm{E}$. Pommier, «Sauver le phénomène éthique pour préserver l'avenir. Jonas et Patočka ou la responsabilité et le sacrifice ", Archives de philosophie, 2016/3 ( $\left.n^{\circ} 79\right)$, p. 525-537.

18. Cf. G. Simondon, L'individuation à la lumière des notions de forme et d'information, Grenoble, Millon, 2005. Nous renvoyons également à S. Margairaz, « Entre apeiron présocratique 
et métastabilité thermodynamique : l'idée de préindividuel chez Gilbert Simondon ", Methodos, 2013 (13).

19. H. Bergson, L'évolution créatrice, op. cit., p. 266.

20. Idem.

21. Idem.

22. Idem.

23. Cf. E. Husserl, La crise de l'humanité européenne et la philosophie, trad. par N. Depraz, Paris, Hatier, 1992, p. 79-80: «Nous posons la question de savoir comment se caractérise la figure spirituelle de l'Europe. L'Europe n'est pas comprise de manière géographique, cartographique, comme si donc le cercle des hommes vivant ici ensemble sur un territoire devait être circonscrit comme humanité européenne ».

24. M. Scheler, Situation de l'homme dans le monde, trad. par M. Dupuis, Paris, Aubier, 1951, p. 89-90.

25. M. Scheler, op. cit., p. 53-54.

26. Cf. M. Bernard, Patočka et l'unité polémique du monde, Louvain, Peeters, 2016.

27. Cf. M. Foucault, L'archéologie du savoir, Paris, Gallimard, 1969, III, chapitre V, "L'a priori historique et l'archive ", p. 173-181.

28. T. W. Adorno, Dialectique négative, trad. par le groupe de traduction du collège de philosophie, Paris, Payot \& Rivages, 2001.

29. Cf. M. Horkheimer et T. W. Adorno, Dialectique de la raison, trad. par E. Kaufholz, Gallimard, 1974 , p. 27 : «La raison se comporte à l'égard des choses comme un dictateur à l'égard des hommes : il les connaît dans la mesure où il peut les manipuler [...]. La nature des choses se révèle [être] toujours la même: le substrat de la domination». C'est en ce sens que «la Raison est totalitaire » (Ibid., p. 24). 